\title{
Correlation Between Collimation-Corrected Peak Luminosity and Spectral Lag of Gamma-ray Bursts in the Source Frame
}

\author{
Heon-Young Chang ${ }^{\dagger}$ \\ Department of Astronomy and Atmospheric Sciences, Kyungpook National University, Daegu 702-701, Korea
}

We revisit the relation between the peak luminosity $L_{\text {iso }}$ and the spectral time lag in the source frame. Since gamma-ray bursts (GRBs) are generally thought to be beamed, it is natural to expect that the collimation-corrected peak luminosity may well correlate with the spectral time lag in the source frame if the lag-luminosity relation in the GRB source frame exists. With 12 long GRBs detected by the Swift satellite, whose redshift and spectral lags in the source frame are known, we computed $L_{0, \mathrm{H}}$ and $L_{0, \mathrm{~W}}$ using bulk Lorentz factors $\Gamma_{0, \mathrm{H}}$ and $\Gamma_{0, \mathrm{~W}}$ archived in the published literature, where the subscripts $\mathrm{H}$ and $\mathrm{W}$ represent homogeneous and wind-like circumburst environments, respectively. We have confirmed that the isotropic peak luminosity correlates with the spectral time lag in the source frame. We have also confirmed that there is an anti-correlation between the source-frame spectral lag and the peak energy, $E_{\text {peak }}(1+z)$ in the source frame. We have found that the collimation-corrected luminosity correlates in a similar way with the spectral lag, except that the correlations are somewhat less tight. The correlation in the wind density profile seems to agree with the isotropic peak luminosity case better than in the homogeneous case. Finally we conclude by briefly discussing its implications.

Keywords: gamma-ray bursts, data analysis

\section{INTRODUCTION}

Gamma-ray bursts (GRBs) are cosmic explosions, which are extremely energetic events occurring in a very short time, and produce highly diverse light curves. GRBs form two distinct populations: the short and long GRBs, defined on the basis of the burst duration (Kouveliotou et al. 1993). Short GRBs are distinguished from long GRBs not only by their duration, but also by various observed properties. For instance, as opposed to long GRBs, for which the isotropic equivalent gamma-ray energy is of the order of $10^{53} \mathrm{erg}$ and for which the host galaxies are typically dwarf galaxies with high star formation rate (Chang 2006, Fruchter et al. 2006, Savaglio et al. 2009), short GRBs are typically less energetic (of the order of $10^{49}-10^{51} \mathrm{erg}$ ) and occur in both earlyand late-type galaxies (Nakar 2007, Berger 2009, 2011). Negligible spectral lag and hard spectra are also common for short GRBs.

Several empirical correlations between various properties of the light curves in prompt gamma-ray emissions and GRB energetics have been discovered. Fenimore \& Ramirez-Ruiz (2000) have found that variable GRBs are much brighter than the smoother ones. Reichart et al. (2001) present a Cepheid-like luminosity estimator based on their finding that the isotropic equivalent peak luminosities positively correlate with a rigorously constructed measure of the variability. Long GRBs with spectroscopically measured redshifts show a correlation between the total isotropic energy and the peak energy of their spectrum (Amati et al. 2002), which is later confirmed with the collimated jet model (Ghirlanda et al. 2004). Yonetoku et al. (2004) have established a relation between the spectral peak energy $\left(E_{\text {peak }}\right)$ and the isotropic peak luminosity $\left(L_{\text {iso }}\right)$. The correlations involving the long GRB prompt emission energy provide a new key to understand the GRB physics. These correlations have also been suggested that long GRBs can be a new class of standard candles in a cosmological distance scale. (c) This is an open Access article distributed under the terms of the Creative Commons Attribution Non-Commercial License (http:// creativecommons.org/licenses/by-nc/3.0/) which premits unrestricted non-commercial use, distribution, and reproduction in any medium, provided the original work is properly cited.
Received May 17, 2012 Revised Jun 7, 2012 Accepted Jun 11, 2012 †Corresponding Author

E-mail: hyc@knu.ac.kr

Tel: +82-53-950-6367 Fax: +82-53-950-6359 
There is another relation which calls for a physical interpretation (Dermer 1998, Ioka \& Nakamura 2001). That is, the spectral time lag has been shown to correlates with the isotropic peak luminosity for long duration bursts (Norris et al. 2000, Norris 2002, Gehrels et al. 2006, Schaefer 2007, Hakkila et al. 2008, Ukwatta et al. 2011). Spectral lag is defined as the difference in time of arrival of high and low energy photons. Recently, Margutti et al. (2010) investigated spectral lags of X-ray flares and found that X-ray flares of long GRBs also exhibit the lag-luminosity correlation observed in the prompt emission. Most of the previous investigations, however, used lags extracted in the observerframe only until Ukwatta et al. $(2010,2012)$ have recently presented an analysis of the lag-luminosity relation in the GRB source frame based on a sample of Swift long GRBs with measured spectroscopic redshifts, $L \propto \tau^{\mathrm{a}}$. Their analysis indicates a higher degree of correlation $-0.82 \pm 0.05$ between spectral lag and the isotropic peak luminosity, $L_{\text {iso }}$, with a best-fit power-law index of $1.2 \pm 0.2$. In addition, an anticorrelation between the source-frame spectral lag and the peak energy in the source frame is also reported.

In this study, we revisit the analysis of Ukwatta et al. (2012) using the collimation-corrected peak luminosity instead of the isotropic one, in order to check if the jet model may conform the relation or even yield a more robust correlation. The compactness problem requires that GRBs are relativistic sources. From this argument lower limits of bulk Lorentz factor $\Gamma_{0}$ of the fireball are usually derived greater than of order of 100 (Lithwick \& Sari 2001). Large Lorentz factors imply strong beaming of the radiation, i.e., relativistic Doppler beaming effect. The observational evidence supporting this idea is the achromatic break of the afterglow light curve, which declines more steeply than in the spherical case (Rhoads 1997, Sari et al. 1999, Panaitescu \& Kumar 2001, Frail et al. 2003). The possibility that GRB fireballs are collimated was first proposed for GRB 970508 (Waxman et al. 1998). Hence, if this is the case, it seems natural that the jet should play a crucial role in determining such a relation.

To tackle this issue, we study here two cases of $\Gamma_{0}$ estimated from the shape of the light curve in two different density profiles, i.e., homogeneous inter-stellar medium (ISM) and varying ISM in density. Generically, a circumburst density profile is described by $n(r)=n_{0}$. While a wind density profile of $n(r)=n * r^{-2}$ is expected from a massive star progenitor that undergoes strong wind mass losses during the final stages of its life (Chevalier \& Li 1999). Even though, it is not possible at the present stage to conclusively prefer the wind ISM to the homogeneous ISM case, Nava et al. (2006) showed, with 18 long GRBs, that the collimation-corrected
$E_{\text {peak }}-E$ correlation (so called 'Ghirlanda' correlation) has a smaller scatter and a linear slope when computed under the assumption of the $\mathrm{W}$ compared to the $\mathrm{H}$ case. It is also, therefore, important to compare the estimates of $\Gamma_{0}$ and further the comoving frame energetics in these two possible scenarios.

\section{SPECTRAL LAG IN THE SOURCE FRAME AND DATA}

The observed spectral lag is extracted between two arbitrary energy bands in the observer frame in the first place. There are three well known ways of extracting spectral lags: pulse peak-fit method (Norris et al. 2005, Hakkila et al. 2008), Fourier analysis method (Li et al. 2004), and crosscorrelation function analysis method (Cheng et al. 1995, Band 1997). These two energy bands can correspond to a different pair of energy bands in the GRB source frame due to the cosmological redshift. Therefore, two corrections are required: 1) correct for the time dilation effect by multiplying the extracted lag value (in the observer frame) by $(1+z)^{-1}(z$-correction), and 2$)$ take into account the fact that for GRBs with various redshifts, observed energy bands correspond to different energy bands at the GRB source frame (K-correction). The second correction is not so straightforward. Gehrels et al. (2006) attempted to approximately correct the spectral lag by multiplying the lag value in the observer frame by $(1+z)^{0.33}$. The approximate $\mathrm{K}$-correction is based on the assumption that spectral lag is proportional to the pulse width and the pulse width is proportional to the energy (Fenimore et al. 1995, Zhang et al. 2009). An alternative is to make the K-correction by defining two energy bands in the GRB source frame to project those two bands into the observer frame and extract lags between them using the relation $E_{\text {observer }}=E_{\text {source }} /(1+z)$, as in Ukwatta et al. $(2010,2012)$. They selected two sourceframe energy bands (100-200 keV and 300-400 keV) of Swift data to extract lags.

In this paper we present a study of spectral lags using a subset of Swift burst alert telescope data. The launch of the Swift satellite (Gehrels et al. 2004) ushered in a new era of GRB research. Since we want to study the energetics, and spectral lags of GRBs in the source frame, our first requirement is to know the redshift $z$. The second selection criterion of GRBs is that the bulk Lorentz factor $\Gamma_{0}$ is known, which can be calculated using the measured peak time of their afterglow light curves. We adopt two possible scenarios, as mentioned above, for the estimate of $\Gamma_{0}$ : the case of a homogeneous circumburst medium $(\mathrm{H})$ or a wind 
Table 1. Sample of GRBs.

\begin{tabular}{lccccrc}
\hline \multicolumn{1}{c}{ GRB } & redshift & lag $(\mathbf{m s})$ & $\begin{array}{c}\boldsymbol{E}_{\text {peak }} \\
(\mathbf{k e V})\end{array}$ & $\boldsymbol{L}_{\text {iso }}(\mathrm{erg} / \mathrm{sec})$ & $\Gamma_{\boldsymbol{0}, H}$ & $\Gamma_{o, W}$ \\
\hline GRB050922C & 2.199 & $136 \pm 68$ & 133 & $5.17 \times 10^{52}$ & 138 & 55 \\
GRB060210 & 3.913 & $658 \pm 259$ & 207 & $8.53 \times 10^{52}$ & 133 & 77 \\
GRB060904B & 0.703 & $124 \pm 436$ & 103 & $2.18 \times 10^{51}$ & 50 & 18 \\
GRB061007 & 1.262 & $52 \pm 22$ & 498 & $1.01 \times 10^{53}$ & 215 & 121 \\
GRB061121 & 1.315 & $22 \pm 10$ & 606 & $7.89 \times 10^{52}$ & 88 & 54 \\
GRB071010B & 0.947 & $404 \pm 159$ & 52 & $4.24 \times 10^{51}$ & 105 & 40 \\
GRB080319C & 1.949 & $174 \pm 91$ & 307 & $6.96 \times 10^{52}$ & 109 & 57 \\
GRB090618 & 0.540 & $267 \pm 72$ & 156 & $8.47 \times 10^{51}$ & 158 & 80 \\
GRB091024 & 1.091 & $912 \pm 604$ & 500 & $5.56 \times 10^{51}$ & 59 & 66 \\
GRB100621A & 0.542 & $1,199 \pm 311$ & 95 & $2.55 \times 10^{51}$ & 26 & 18 \\
GRB100906A & 1.727 & $105 \pm 79$ & 180 & $4.90 \times 10^{52}$ & 186 & 93 \\
GRB110213A & 1.460 & $602 \pm 746$ & 98 & $3.53 \times 10^{51}$ & 113 & 51 \\
\hline
\end{tabular}

Columns 1 and 2 show the gamma-ray burst (GRB) name and its redshift, column 3 the extracted spectral lags in ms, column 4 the rest frame peak energy $\mathrm{E}_{\text {peak }}$ in $\mathrm{eV}$, and column 5 the isotropic equivalent luminosity $L_{\text {iso }}$. In columns 6 and 7 we show the $\Gamma_{0}$ factor in the $H$ case and in the $W$ case, respectively.

density profile (W). We have searched in the literature for this criterion and taken them from Ghirlanda et al. (2012). For spectral lags, thirdly, we have taken data of GRBs from Ukwatta et al. (2010, 2012). Finally, we only choose GRBs whose spectral lag is positive, i.e., only those in whose light curves the high-energy photons arrive earlier than the low energy ones.

The spectral information for the 12 bursts used in this paper is given in Table 1. Columns 1 and 2 show the GRB name and its redshift, column 3 the extracted spectral lags in ms, column 4 the rest frame peak energy $E_{\text {peak }}$ in $\mathrm{keV}$, and column 5 the isotropic equivalent luminosity $L_{\text {iso }}$. In columns 6 and 7 we show the $\Gamma_{0}$ factor in the $H$ case (column 6 ) and in the $\mathrm{W}$ case (column 7 ) assuming a typical density value $n_{0}=3 \mathrm{~cm}^{-3}$ and a typical radiative efficiency $\eta=0.2$. This sample used in our analysis has redshifts ranging from 0.540 (GRB 090618) to 3.913 (GRB 060210) with an average redshift of 1.467 . The values of $\Gamma_{0}$ are broadly distributed between few tens and several hundreds with average values 115 and 60 for the homogeneous and wind density profiles, respectively.

\section{RESULTS AND DISCUSSION}

In Fig. 1, we show the source-frame peak energy $E_{\text {peak }}$ versus spectral lag for $12 \mathrm{GRBs}$ in log-log plot. The straight line represents the best-fit of data obtained by the linear least-squares method. As reported earlier, there is an anticorrelation between the source-frame spectral lag and the peak energy, $E_{\text {peak }}(1+z)$ in the source frame. The Pearson's correlation coefficient for this relation is -0.55 with a chance probability of 0.033 . The best-fit power-law index is $-0.39 \pm$
Table 2. Correlation coefficients of the lag - $E_{\text {peak }}$ relation.

\begin{tabular}{lcc}
\hline Coefficient type & Correlation coefficient & Null probability \\
\hline Pearson's $r$ & -0.5452 & 0.0333 \\
Spearman's $r_{s}$ & -0.5734 & 0.0256 \\
Kendall's $\tau$ & -0.4242 & 0.0548 \\
\hline
\end{tabular}

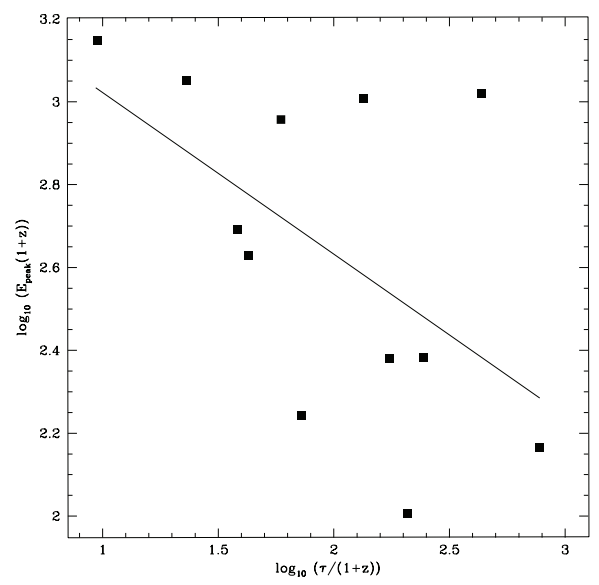

Fig. 1. Source frame peak energy $E_{\text {peak }}$ versus spectral lag. The straight line represents the best-fit of data obtained by the linear least-squares method. The uncertainty in the slope is 0.25 .

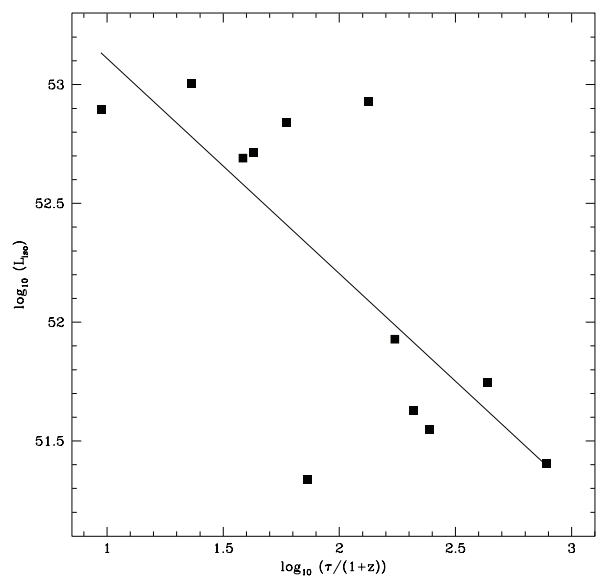

Fig. 2. Source frame peak luminosity $L_{\text {iso }}$ versus spectral lag. The uncertainty in the slope is 0.33 .

0.19. For comparison with results of Ukwatta et al. (2012), they reported the Pearson's coefficient of $-0.57 \pm 0.14$ for the 43 long GRBs, which can be considered as a parent sample of ours. We provide various correlation coefficient of the relations with null probabilities in Table 2.

In Fig. 2, we show the source-frame isotropic peak luminosity $L_{\text {iso }}$ versus spectral lag in log-log plot. The straight line is the best-fit of data. The Pearson's correlation coefficient for this relation is -0.75 with a null hypothesis 


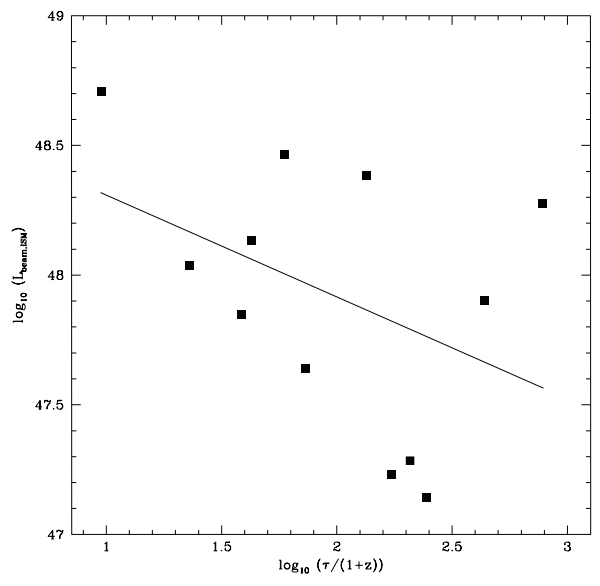

Fig. 3. Source frame peak luminosity $L_{0, H}$ versus spectral lag. The uncertainty in the slope is 0.29 .

Table 3. Correlation coefficients of the lag $-L_{\text {iso }}$ relation.

\begin{tabular}{lcc}
\hline Coefficient type & Correlation coefficient & Null probability \\
\hline Pearson's $r$ & -0.7453 & 0.0027 \\
Spearman's $r_{s}$ & -0.6923 & 0.0062 \\
Kendall's $\tau$ & -0.5151 & 0.0197 \\
\hline
\end{tabular}

Table 4. Correlation coefficients of the lag $-L_{0, H}$ relation.

\begin{tabular}{lcc}
\hline Coefficient type & Correlation coefficient & Null probability \\
\hline Pearson's $r$ & -0.4244 & 0.0845 \\
Spearman's $r_{s}$ & -0.4055 & 0.0954 \\
Kendall's $\tau$ & -0.3030 & 0.1702 \\
\hline
\end{tabular}

Table 5. Correlation coefficients of the lag $-L_{0, w}$ relation

\begin{tabular}{lcc}
\hline Coefficient type & Correlation coefficient & Null probability \\
\hline Pearson's $r$ & -0.6169 & 0.0162 \\
Spearman's $r_{s}$ & -0.5805 & 0.0239 \\
Kendall's $\tau$ & -0.4545 & 0.0396 \\
\hline
\end{tabular}

probability of being uncorrelated of 0.0027 (for the correlation coefficients, e.g., see Press et al. [1992]). The best-fit power-law index is $-0.91 \pm 0.26$. The extracted correlation coefficient is compatible with the correlation coefficient of $-0.82 \pm 0.05$ reported in Ukwatta et al. (2012). According to Figs. 1 and 2 we consider our sample is hardly biased, even though GRBs are selected according to the criteria we provided in the last section. Various correlation coefficient of the relations with null probabilities are provided in Table 3.

In Figs. 3 and 4, we show the source-frame peak luminosity $L_{0, \mathrm{H}}$ and $L_{0, \mathrm{~W}}$ versus spectral lag in log-log plot, respectively. The subscripts $H$ and $W$ represent homogeneous and wind-like circumburst environments, respectively. We computed collimated peak luminosity

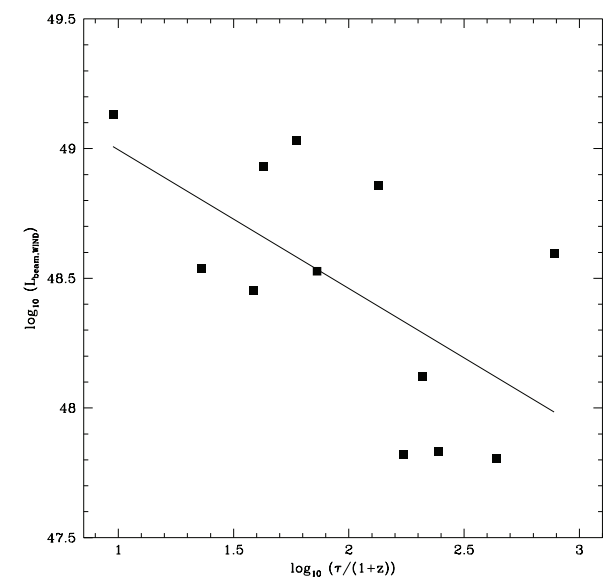

Fig. 4. Source frame peak luminosity $L_{0, w}$ versus spectral lag. The uncertainty in the slope is 0.24 .

using $\Gamma_{0, \mathrm{H}}$ and $\Gamma_{0, \mathrm{w}}$ in Table 1. The straight lines are the bestfits of data. The Pearson's correlation coefficients for these relations are -0.42 and -0.61 with chance probabilities of 0.08 and 0.001 , respectively. The best-fit power-law indices are $-0.39 \pm 0.26$ and $-0.53 \pm 0.21$, respectively. We provide various correlation coefficients of the relation with null probabilities in Tables 4 and 5. We find that the collimationcorrected luminosity correlates in a similar way with spectral lag, except that the correlations are somewhat less tight. The correlation in the wind density profile seems to agree with the isotropic peak luminosity case better than in the homogeneous case. There are two important changes in the lag-luminosity relation which may occur when going from isotropic energy to collimated energy in the source frame, i.e., a change in the powerlaw index, and a change in the correlation coefficient. This correlation can shed light on the still uncertain radiation processes for the prompt GRB emission (Nava et al. 2006).

\section{DISCUSSION AND CONCLUSIONS}

Using a sample of 43 Swift GRBs, Ukwatta et al. (2012) found that the correlation coefficient improves significantly in the source frame. Since GRBs are generally thought to be beamed, it is natural to expect that the collimationcorrected peak luminosity may well correlate with the spectral time lag in the source frame if the lag-luminosity relation in the GRB source frame exists. We revisit the analysis of Ukwatta et al. (2012) using the collimationcorrected peak luminosity instead of the isotropic one. Using 12 long GRBs detected by the Swift satellite, whose redshift and spectral lags in the source frame are known, we 
derived $L_{0, \mathrm{H}}$ and $L_{0, \mathrm{~W}}$ using bulk Lorentz factors $\Gamma_{0, \mathrm{H}}$ and $L_{0, \mathrm{~W}}$ archived in the published literature. By doing so, one may expect an extension from Ukwatta relation to another, such as, one from Amati relation to Ghirlanda relation.

We have found that our sample is hardly biased, since correlation coefficients for the relation between the sourceframe peak luminosity $L_{\text {iso }}$ and spectral lag are compatible with the correlation coefficients reported in Ukwatta et al. (2012). We have also confirmed that there is an anticorrelation between the source-frame spectral lag and the peak energy, $E_{\text {peak }}(1+z)$ in the source frame. In the present analysis we have found that the collimation-corrected luminosity correlates in a similar way with spectral lag, except that the correlations are somewhat less tight. The correlation in the wind density profile seems to agree with the isotropic peak luminosity case better than in the homogeneous case. This finding corresponds to earlier report by Nava et al. (2006) that the collimation-corrected $E_{\text {peak }}-E$ correlation has a smaller scatter when computed under the assumption of the $\mathrm{W}$ compared to the $\mathrm{H}$ case. Even if several ideas have been already discussed in the literature, this correlation would contribute to understand the underlying radiation process of the prompt emission of GRBs in the source frame.

\section{ACKNOWLEDGMENTS}

HYC was supported by the National Research Foundation of Korea Grant funded by the Korean government (NRF2011-0008123) and also thanks the anonymous referees for critical comments and helpful suggestions which greatly improve the original version of the manuscript.

\section{REFERENCES}

Amati L, Frontera F, Tavani M, in't Zand JJM, Antonelli A, et al., Intrinsic spectra and energetics of BeppoSAX gamma-ray bursts with known redshifts, A\&A, 390, 81-89 (2002). http://dx.doi.org/10.1051/00046361:20020722

Band DL, Gamma-ray burst spectral evolution through cross-correlations of discriminator light curves, ApJ, 486, 928-937 (1997). http://dx.doi.org/10.1086/304566

Berger $\mathrm{E}$, The host galaxies of short-duration gammaray bursts: luminosities, metallicities, and starformation rates, ApJ, 690, 231-237 (2009). http://dx.doi. org/10.1088/0004-637X/690/1/231

Berger $\mathrm{E}$, The environments of short-duration gamma- ray bursts and implications for their progenitors, NewAR, 55, 1-22 (2011). http://dx.doi.org/10.1016/ j.newar.2010.10.001

Chang H-Y, The peak energy-duration correlation and possible implications on gamma ray burst progenitor, JASS, 23, 167-176 (2006). http://dx.doi.org/10.5140/ JASS.2006.23.3.167

Cheng LX, Ma YQ, Cheng KS, Lu T, Zhou YY, The time delay of gamma-ray bursts in the soft energy band, A\&A, 300, 746-750 (1995).

Chevalier RA, Li Z-Y, Gamma-ray burst environments and progenitors, ApJ, 520, L29-L32 (1999). http://dx.doi. org/10.1086/312147

Dermer CD, On spectral and temporal variability in blazars and gamma-ray bursts, ApJ, 501, L157-L160 (1998). http://dx.doi.org/10.1086/311467

Fenimore EE, in't Zand JJM, Norris JP, Bonnell JT, Nemiroff RJ, Gamma-ray burst peak duration as a function of energy, ApJ, 448, L101-L104 (1995). http://dx.doi. org/10.1086/309603

Fenimore EE, Ramirez-Ruiz E, Redshifts for 220 BATSE gamma-ray bursts determined by variability and the cosmological consequences (2000). http://arxiv.org/ abs/astro-ph/0004176v2

Frail DA, Kulkarni SR, Berger E, Wieringa MH, A complete catalog of radio afterglows: the first five years, AJ, 125, 2299-2306 (2003). http://dx.doi.org/10.1086/374364

Fruchter AS, Levan AJ, Strolger L, Vreeswijk PM, Thorsett SE, et al., Long gamma-ray bursts and core-collapse supernovae have different environments, Natur, 441, 463-468 (2006). http://dx.doi.org/10.1038/nature04787

Gehrels N, Chincarini G, Giommi P, Mason KO, Nousek JA, et al., The swift gamma-ray burst mission, ApJ, 611, 1005-1020 (2004). http://dx.doi.org/10.1086/422091

Gehrels N, Norris JP, Barthelmy SD, Granot J, Kaneko Y, et al., A new gamma-ray burst classification scheme from GRB060614, Natur, 444, 1044-1046 (2006). http://dx.doi. org/10.1038/nature05376

Ghirlanda G, Ghisellini G, Lazzati D, The collimationcorrected GRB energies correlate with the peak energy of their $v \mathrm{~F}_{v}$ spectrum, ApJ, 616, 331-338 (2004). http:// dx.doi.org/10.1086/424913

Ghirlanda G, Nava L, Ghisellini G, Celotti A, Burlon D, et al., Gamma-ray bursts in the comoving frame, MNRAS, 420, 483-494 (2012). http://dx.doi.org/10.1111/j.13652966.2011.20053.x

Hakkila J, Giblin TW, Norris JP, Fragile PC, Bonnell JT, Correlations between lag, luminosity, and duration in gamma-ray burst pulses, ApJ, 677, L81-L84 (2008). http://dx.doi.org/10.1086/588094 
Ioka K, Nakamura T, Peak luminosity-spectral lag relation caused by the viewing angle of the collimated gammaray bursts, ApJ, 554, L163-L167 (2001). http://dx.doi. org/10.1086/321717

Kouveliotou C, Meegan CA, Fishman GJ, Bhat NP, Briggs MS, et al., Identification of two classes of gammaray bursts, ApJ, 413, L101-L104 (1993). http://dx.doi. org/10.1086/186969

Li T-P, Qu J-L, Feng H, Song L-M, Ding G-Q, et al., Timescale analysis of spectral lags, ChJAA, 4, 583-598 (2004).

Lithwick Y, Sari R, Lower limits on lorentz factors in gammaray bursts, ApJ, 555, 540-545 (2001). http://dx.doi. org/10.1086/321455

Margutti R, Guidorzi C, Chincarini G, Bernardini MG, Genet F, et al., Lag-luminosity relation in gammaray burst X-ray flares: a direct link to the prompt emission, MNRAS, 406, 2149-2167 (2010). http://dx.doi. org/10.1111/j.1365-2966.2010.16824.x

Nakar E, Short-hard gamma-ray bursts, PhR, 442, 166-236 (2007). http://dx.doi.org/10.1016/j.physrep.2007.02.005

Nava L, Ghisellini G, Ghirlanda G, Tavecchio F, Firmani C, On the interpretation of spectral-energy correlations in long gamma-ray bursts, A\&A, 450, 471-481 (2006). http://dx.doi.org/10.1051/0004-6361:20054211

Norris JP, Implications of the lag-luminosity relationship for unified gamma-ray burst paradigms, ApJ, 579, 386-403 (2002). http://dx.doi.org/10.1086/342747

Norris JP, Bonnell JT, Kazanas D, Scargle JD, Hakkila J, et al., Long-lag, wide-pulse gamma-ray bursts, ApJ, 627, 324345 (2005). http://dx.doi.org/10.1086/430294

Norris JP, Marani GF, Bonnell JT, Connection between energy-dependent lags and peak luminosity in gammaray bursts, ApJ, 534, 248-257 (2000). http://dx.doi. org/10.1086/308725

Panaitescu A, Kumar P, Fundamental physical parameters of collimated gamma-ray burst afterglows, ApJ, 560, L49L53 (2001). http://dx.doi.org/10.1086/324061

Press WH, Flannery BP, Teukolsky SA, Vetterling WT, Numerical recipes in FORTRAN (Cambridge University Press, Cambridge, 1992).

Reichart DE, Lamb DQ, Fenimore EE, Ramirez-Ruiz E, Cline $\mathrm{TL}$, et al., A possible cepheid-like luminosity estimator for the long gamma-ray bursts, ApJ, 552, 57-71 (2001). http://dx.doi.org/10.1086/320434

Rhoads JE, How to tell a jet from a balloon: a proposed test for beaming in gamma-ray bursts, ApJ, 487, L1-L4 (1997). http://dx.doi.org/10.1086/310876

Sari R, Piran T, Halpern JP, Jets in gamma-ray bursts, ApJ, 519, L17-L20 (1999). http://dx.doi.org/10.1086/312109

Savaglio S, Glazebrook K, Le Borgne D, The galaxy population hosting gamma-ray bursts, ApJ, 691, 182-211 (2009). http://dx.doi.org/10.1088/0004$637 \mathrm{X} / 691 / 1 / 182$

Schaefer BE, The Hubble diagram to redshift $>6$ from 69 gamma-ray bursts, ApJ, 660, 16-46 (2007). http://dx.doi. org/10.1086/511742

Ukwatta TN, Dhuga KS, Morris DC, MacLachlan G, Parke WC, et al., A new frequency-luminosity relation for long gamma-ray bursts?, MNRAS, 412, 875-882 (2011). http://dx.doi.org/10.1111/j.1365-2966.2010.17944.x

Ukwatta TN, Dhuga KS, Stamatikos M, Dermer CD, Sakamoto T, et al., The lag-luminosity relation in the GRB source frame: an investigation with Swift BAT bursts, MNRAS, 419, 614-623 (2012). http://dx.doi. org/10.1111/j.1365-2966.2011.19723.x

Ukwatta TN, Stamatikos M, Dhuga KS, Sakamoto T, Barthelmy SD, et al., Spectral lags and the lagluminosity relation: an investigation with swift BAT gamma-ray bursts, ApJ, 711, 1073-1086 (2010). http:// dx.doi.org/10.1088/0004-637X/711/2/1073

Waxman E, Kulkarni SR, Frail DA, Implications of the radio afterglow from the gamma-ray burst of 1997 May 8, ApJ, 497, 288-293 (1998). http://dx.doi.org/10.1086/305467

Yonetoku D, Murakami T, Nakamura T, Yamazaki R, Inoue AK, et al., Gamma-ray burst formation rate inferred from the spectral peak energy-peak luminosity relation, ApJ, 609, 935-951 (2004). http://dx.doi. org/10.1086/421285

Zhang B, Zhang B-B, Virgili FJ, Liang E-W, Kann DA, et al., Discerning the physical origins of cosmological gammaray bursts based on multiple observational criteria: the cases of $\mathrm{z}=6.7$ GRB 080913, $\mathrm{z}=8.2$ GRB 090423, and some short/hard GRBs, ApJ, 703, 1696-1724 (2009). http://dx.doi.org/10.1088/0004-637X/703/2/1696 